\title{
Time to positivity in blood cultures of adults with Streptococcus
} pneumoniae bacteremia

\author{
Galo Peralta*1, María José Rodríguez-Lera², Jose Carlos Garrido3, \\ Luis Ansorena ${ }^{4}$ and María Pía Roiz ${ }^{5}$
}

\begin{abstract}
Address: ${ }^{1}$ Internal Medicine Service, Sierrallana Hospital, Barrio de Ganzo s/n, 39120 Torrelavega, Cantabria, Spain, ${ }^{2}$ Emergency Service, Sierrallana Hospital, Barrio de Ganzo s/n, 39120 Torrelavega, Cantabria, Spain, ${ }^{3}$ Laboratory Service, Sierrallana Hospital, Barrio de Ganzo s/n, 39120 Torrelavega, Cantabria, Spain, ${ }^{4}$ Admission Service, Sierrallana Hospital, Barrio de Ganzo s/n, 39120 Torrelavega, Cantabria, Spain and ${ }^{5}$ Microbiology Service, Sierrallana Hospital, Barrio de Ganzo s/n, 39120 Torrelavega, Cantabria, Spain

Email: Galo Peralta* - gpf@mundivia.es; María José Rodríguez-Lera - mrodriguezl@hsll.scsalud.es; Jose Carlos Garrido - jgarrido@hsll.scsalud.es; Luis Ansorena - lansorena@hsll.scsalud.es; María Pía Roiz - mproiz@yahoo.es

* Corresponding author
\end{abstract}

Published: 27 April 2006

BMC Infectious Diseases 2006, 6:79 doi:10.1 186/1471-2334-6-79
Received: 17 November 2005

Accepted: 27 April 2006

This article is available from: http://www.biomedcentral.com/147I-2334/6/79

(C) 2006 Peralta et al; licensee BioMed Central Ltd.

This is an Open Access article distributed under the terms of the Creative Commons Attribution License (http://creativecommons.org/licenses/by/2.0), which permits unrestricted use, distribution, and reproduction in any medium, provided the original work is properly cited.

\begin{abstract}
Background: previous studies have established that bacterial blood concentration is related with clinical outcome. Time to positivity of blood cultures (TTP) has relationship with bacterial blood concentration and could be related with prognosis. As there is scarce information about the usefulness of TTP, we study the relationship of TTP with clinical parameters in patients with Streptococcus pneumoniae bacteremia.
\end{abstract}

Methods: TTP of all cases of Streptococcus pneumoniae bacteremia, detected between January 1995 and December 2004 using the BacT/Alert automated blood culture system in a teaching community hospital was analyzed. When multiple cultures were positive only the shortest TTP was selected for the analysis.

Results: in the study period 105 patients with Streptococcus pneumoniae bacteremia were detected. Median TTP was I4.I hours (range I.2 h to $127 \mathrm{~h}$ ). Immunosuppressed patients $(\mathrm{n}=5)$, patients with confusion $(n=19)$, severe sepsis or shock at the time of blood culture extraction $(n=12)$, those with a diagnosis of meningitis $(n=7)$ and those admitted to the ICU $(n=14)$ had lower TTP. Patients with TTP in the first quartile were more frequently hospitalized, admitted to the ICU, had meningitis, a non-pneumonic origin of the bacteremia, and a higher number of positive blood cultures than patients with TTP in the fourth quartile. None of the patients with TTP in the $90^{\text {th }}$ decile had any of these factors associated with shorter TTP, and eight out of ten patients with TTP in the $10^{\text {th }}$ decile had at least one of these factors. The number of positive blood cultures had an inverse correlation with TTP, suggesting a relationship of TTP with bacterial blood concentration.

Conclusion: Our data support the relationship of TTP with several clinical parameters in patients with Streptococcus pneumoniae bacteremia, and its potential usefulness as a surrogate marker of outcome. 


\section{Background}

Streptococcus pneumoniae is a major cause of pneumonia, meningitis and bacteremia and is among the leading infectious causes of illness and death worldwide [1]. The mortality of pneumococcal bacteremia remains high and is related to several prognostic factors [2-7]. The time to positivity of blood cultures (TTP) of patients with Streptococcus pneumoniae bacteremia has previously been explored as a marker of outcome, but no relationship with clinical or laboratory parameters has been found in children [8]. TTP of simulated blood cultures with Staphylococcus epidermidis has a linear correlation with initial bacterial concentration $[9,10]$ although this has not studied with Streptococcus pneumoniae. In addition, several researchers have found a correlation between concentration of bacteria in blood and the presence of meningitis and its prognosis [11-16]. Because it is unknown if TTP correlates with outcome we decide to explore its relationship with clinical parameters and prognosis in adult patients with Streptococcus pneumoniae bacteremia.

\section{Methods}

Patients

The study was conducted at the Sierrallana Hospital, a 220-bed community hospital. All the blood cultures taken from January 1995 to December 2004 were reviewed and patients whose blood cultures yielded Streptococcus pneumoniae were selected for the study.

A standardized data collection form was used to review the hospital records of patients with pneumococcal bacteremia. Pneumococcal pneumonia was defined by the presence of acute respiratory symptoms and fever in association with new radiographic infiltrates in a patient with pneumococcal bacteremia. Pneumococcal meningitis was defined by compatible clinical symptoms including headache and meningismus, and growth of Streptococcus pneumoniae or pleocytosis of at least 100 neutrophils per cubic millimeter, in cerebrospinal fluid. Primary bloodstream infection was defined by the documentation of pneumococcal bacteremia in the absence of any recognized local infection, which might have given rise to the bacteremia. Sepsis, severe sepsis, and septic shock were defined according to American College of Chest Physicians and Society of Critical Care Medicine consensus statement [17]. The study has been approved by the local ethics committee.

\section{Blood cultures}

The recommended practice in our hospital is to obtain 20 $\mathrm{mL}$ of venous blood and innoculate it in equal parts into one aerobic (BacT/ALERT FA aerobic, bioMerieux Corporation, Durham, North Carolina) and one anaerobic blood culture bottle (BacT/ALERT FN, bioMerieux). Blood extraction is usually performed three times at intervals of
30 minutes. The blood culture bottles obtained for each patient are immediately sent to the microbiology laboratory and introduced into the blood culture instrument (BacT/ALERT microbial detection system, bioMerieux), which has a colorimetric carbon dioxide sensor to measure microbial growth. The system tests for carbon dioxide production and records the time elapsed from the placement of each blood culture bottle in the system to the detection of microbial growth. This time is considered as the TTP. The bottles are incubated for five days. When multiple cultures were positive only the shortest TTP was selected for the analysis. Streptococcus pneumoniae were identified using standard procedures. Time interval between obtaining the blood for culture and incubation of the bottles was not recorded.

\section{Statistical analysis}

Otherwise indicated data are expressed as mean \pm standard deviation. In statistical analyses, Student's t test (two tailed) and the Mann-Whitney rank-sum test were used for the comparison of mean values, Fisher's exact test and the $\chi^{2}$ test were used for the assessment of proportions, and Sperman's correlation coefficient to explore the correlation between two variables. The statistical analysis was performed using SPSS software, version 12.0.

\section{Results \\ Patients}

One hundred and five patients with Streptococcus pneumoniae blood isolates were identified at the Sierrallana Hospital between January 1, 1995, and December 31, 2004. The overall mean age was 65.9 years (range: $16-93$ years). Forty four patients $(41.9 \%)$ were female. Ninety four patients $(89.5 \%)$ were admitted to hospital and positive blood cultures were obtained from the remaining 11 patients $(10.5 \%)$ at emergency visits, but they were not hospitalized. The mean hospital stay was 13.9 days (range: 1-54 days).

Concurrent pneumonia was present in 85 patients $(81 \%)$, meningitis in seven $(6.7 \%)$, and sinusitis, spontaneous bacterial peritonitis, cholangitis, and hip prosthesis infection, in one patient each. Endocarditis was also diagnosed in one patient with meningitis. No source of bacteremia was identified in 8 patients $(7.6 \%)$. The origin of the bacteremia was nosocomial in only two cases. Eleven patients $(10.5 \%)$ had severe sepsis and one patient met septic shock criteria.

Nine patients $(8.6 \%)$ were on treatment with antibiotics at the moment of the blood culture extraction. Three with oral macrolides, one with an oral and one with a intramuscular cephalosporin, one with oral ciprofloxacin, one with oral ampicillin-sulbactam, and one with a non specified oral antibiotic. 
Five patients had immunosuppression (4.8\%), three due to steroid treatment, one due to HIV infection, and one due to an advanced multiple myeloma. Fourteen patients $(13.3 \%)$ were admitted to the ICU, six because of meningitis, five due to respiratory insufficiency requiring mechanical ventilation, one due to multiorgan failure, one due to digestive haemorrhage, and one due to a supraventricular tachycardia. Of the 13 patients who died, five had bilateral pneumonia, three had severe sepsis and one had septic shock. The cause of death was respiratory failure in ten patients, cardiac failure in two and septic shock in one. Only one of the patients who died had been admitted to the ICU.

\section{Blood cultures}

The number of the blood culture bottles obtained from each patient with pneumococcal bacteremia was: six bottles in 97 patients $(92.4 \%)$, five bottles in one patient, four bottles in two patients, three bottles in one patient, and two bottles in four patients. In one patient whose bacteremia had no apparent origin, Escherichia coli was isolated together with Streptococcus pneumoniae from one blood culture, and its TTP was not considered for the analysis. In another two of the six bottles obtained from this patient, Streptococcus pneumoniae grew alone and the TTP was included in the analysis.

Three Streptococcus pneumoniae isolated from blood cultures had a high-level of penicillin resistance (MIC $\geq 2 \mu \mathrm{g} /$ $\mathrm{mL}$ ) and 17 had intermediate penicillin resistance (MIC between 0.1 and $2 \mu \mathrm{g} / \mathrm{mL}$ ).

\section{Time to positivity}

TTP was available from all patients. All but two patients had a TTP shorter than 22 hours. The two other patients had TTPs of 120 hours and 127 hours. Pneumonia was diagnosed in both patients, who had non-severe sepsis and survived without ICU support.

Median TTP was $14.1 \mathrm{~h}$ (range: 1.2 to $127 \mathrm{~h}$ ) (Figure 1 ). The $25^{\text {th }}$ percentile of TTP was $10.9 \mathrm{~h}$, the $75^{\text {th }}$ percentile was $13.7 \mathrm{~h}$, and the $10^{\text {th }}$ and $90^{\text {th }}$ deciles of TTP were 7.9 $\mathrm{h}$ and $15.4 \mathrm{~h}$ respectively. Among 78 patients with more than one positive blood culture median maximal difference of the TTP among the blood culture bottles of each patient was $1.1 \mathrm{~h}$ (interquartile range $0.67-2.4 \mathrm{~h}$ ) and the median intrapatient TTP variation coefficient was 4.2 (interquartile range 1.9-22.9) (Figure 2). Among 75 patients with aerobes and anaerobes positive blood cultures, in $40(53.3 \%)$ the anaerobes bottles had lower TTP. The median difference among shortest aerobe bottles TTP and anaerobe bottles TTP of each of these patients was $0.08 \mathrm{~h}$ (interquartile range $-0.57-0.33 \mathrm{~h}$ ).

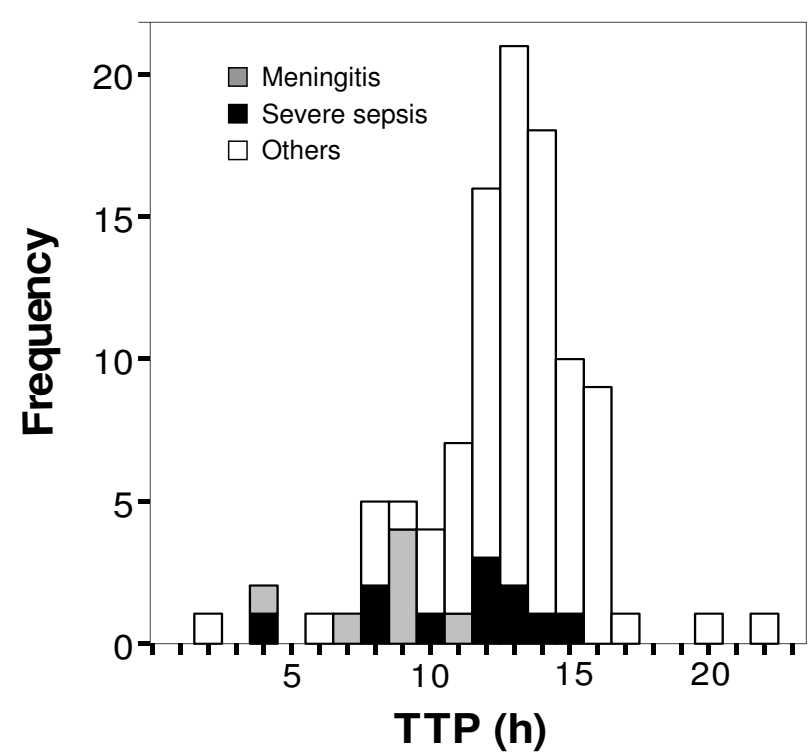

Figure I

TTP in the 105 patients with Streptococcus pneumoniae bacteremia. Two patients with extreme values of TTP are excluded (I $20 \mathrm{~h}$ and $\mathrm{I} 22 \mathrm{~h}$ respectively). One patient in the group of meningitis had also severe sepsis.

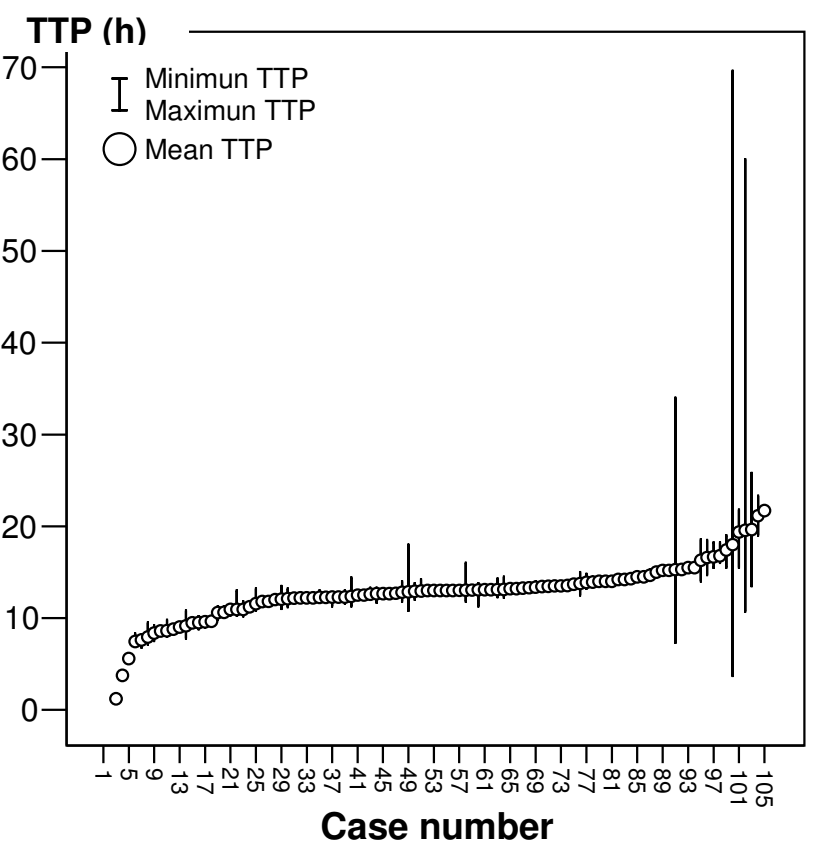

\section{Figure 2}

The minimal, maximal and mean TTP value of the blood cultures of each patient are represented. Two patients with extreme values of TTP are excluded $(120 \mathrm{~h}$ and $122 \mathrm{~h}$ respectively, each only with one positive blood culture). 
No statistically significant differences were detected in TPP depending on sex, age, comorbidities, existence of chills, prior antibiotic treatment, bilateral pneumonia, Streptococcus pneumoniae penicillin susceptibility or fatal outcome. Immunosuppressed patients, those with confusion, severe sepsis or shock at the time of blood culture extraction had statistically significant lower TTP, as well as those diagnosed with meningitis and those admitted to the ICU (Table 1, Figure 3).

When we analyzed the differences between the characteristics of patients with TTP in the first quartile and in the fourth quartile, we detected statistically significant differences between both groups in the proportion of hospitalized patients, number of patients admitted to ICU, and those with meningitis. Patients with TTP in the first quartile also a higher number of positive blood cultures than patients with TTP in the fourth quartile (Table 2).

Eight of the ten patients with TTP in the $10^{\text {th }}$ decile had at least a factor associated with shorter TTP: four had severe sepsis (one with multiorgan failure), two were immuno- suppressed (one treated with steroids and the other diagnosed with multiple myeloma), two were diagnosed with meningitis (one of them also had an endocarditis) and four required a stay in the ICU. None of the patients with TTP in the $90^{\text {th }}$ decile had meningitis, severe sepsis, shock or immunosuppression, nor required ICU admission.

A significant correlation was found between the TTP and the number of positive blood cultures from each episode (Figure 4).

\section{Discussion}

In our patients with Streptococcus pneumoniae bacteremia we found a relationship between TTP and several clinical parameters. TTP is shorter in hospitalized patients, patients with immunosuppression, meningitis, confusion, severe sepsis or shock and in those admitted to ICU. Moreover, most of the patients with the shortest TTP (TTP in the first decile) had a factor that could explain this short TTP. However, a shorter TTP was not detected in the patients who died. The dissociation of TTP between ICU patients and those who died may be attributable to the

Table I: Comparison of TTP values of the 103 patients in relation to clinical parameters.

\begin{tabular}{|c|c|c|c|}
\hline \multirow[t]{2}{*}{ Characteristic (n) } & \multicolumn{2}{|c|}{ TTP } & \multirow[b]{2}{*}{$\mathbf{p}$} \\
\hline & With the factor & Without the factor & \\
\hline \multicolumn{4}{|l|}{ Basal characteristics } \\
\hline Male $(n=6 I)$ & $12.06 \pm 3.04$ & $11.85 \pm 2.84$ & $>0.1$ \\
\hline Older than 75 y $(n=4 I)$ & $11.81 \pm 3.64$ & $12.07 \pm 2.44$ & $>0.1$ \\
\hline Hospitalized $(n=94)$ & $11.79 \pm 3.06$ & $13.42 \pm 1.08$ & 0.001 \\
\hline Previous antibiotic* $(n=9)$ & $11.3 \pm 3.84$ & $12.03 \pm 2.87$ & $>0.1$ \\
\hline Penicillin susceptible $(n=85)$ & $11.77 \pm 2.88$ & $12.8 \pm 3.19$ & $>0.1$ \\
\hline \multicolumn{4}{|l|}{ Comorbidities } \\
\hline Alcoholism (n = 17) & $12.92 \pm 2.48$ & $11.78 \pm 3.01$ & $>0.1$ \\
\hline $\operatorname{COPD}(n=3 I)$ & $12.61 \pm 3.74$ & $11.72 \pm 2.56$ & $>0.07$ \\
\hline Diabetes $(n=I I)$ & $11.77 \pm 1.43$ & $11.99 \pm 3.07$ & $>0.1$ \\
\hline Cirrhosis $(n=7)$ & $11.42 \pm 2.63$ & $12 \pm 2.98$ & $>0.1$ \\
\hline Immunosuppression $(n=5)$ & $9.4 \pm 5.2$ & $12.1 \pm 2.77$ & 0.05 \\
\hline Dementia $(n=6)$ & $1 \mathrm{I} .63 \pm 4.4$ & $11.99 \pm 2.89$ & $>0.1$ \\
\hline \multicolumn{4}{|l|}{ Clinical presentation } \\
\hline Confusion $(n=19)$ & $10.36 \pm 2.58$ & $12.33 \pm 2.68$ & 0.01 \\
\hline Severe sepsis or shock $(n=12)$ & $10.22 \pm 3.05$ & $12.2 \pm 2.87$ & 0.02 \\
\hline Chills $(n=34)$ & $12.17 \pm 2.59$ & $11.88 \pm 3.11$ & $>0.1$ \\
\hline Bilateral pneumonia $(n=13)$ & $12.24 \pm 3.89$ & $11.98 \pm 2.80$ & $>0.1$ \\
\hline \multicolumn{4}{|l|}{ Source of bacteremia } \\
\hline Meningitis $(n=7)$ & $7.81 \pm 2.1$ & $12.27 \pm 2.78$ & $<0.001$ \\
\hline Pneumonia $(n=85)$ & $12.19 \pm 2.71$ & $11.05 \pm 3.72$ & $<0.03$ \\
\hline Primary $(n=8)$ & $13.33 \pm 3.55$ & $11.85 \pm 2.89$ & $>0.1$ \\
\hline \multicolumn{4}{|l|}{ Outcome } \\
\hline $\operatorname{ICU}(n=14)$ & $9.22 \pm 2.48$ & $12.4 \pm 2.79$ & $<0.001$ \\
\hline Exitus $(n=13)$ & $11.40 \pm 4.17$ & $12.05 \pm 2.75$ & $>0.1$ \\
\hline
\end{tabular}

* Including ambulatory treatment immediately before blood culture. 

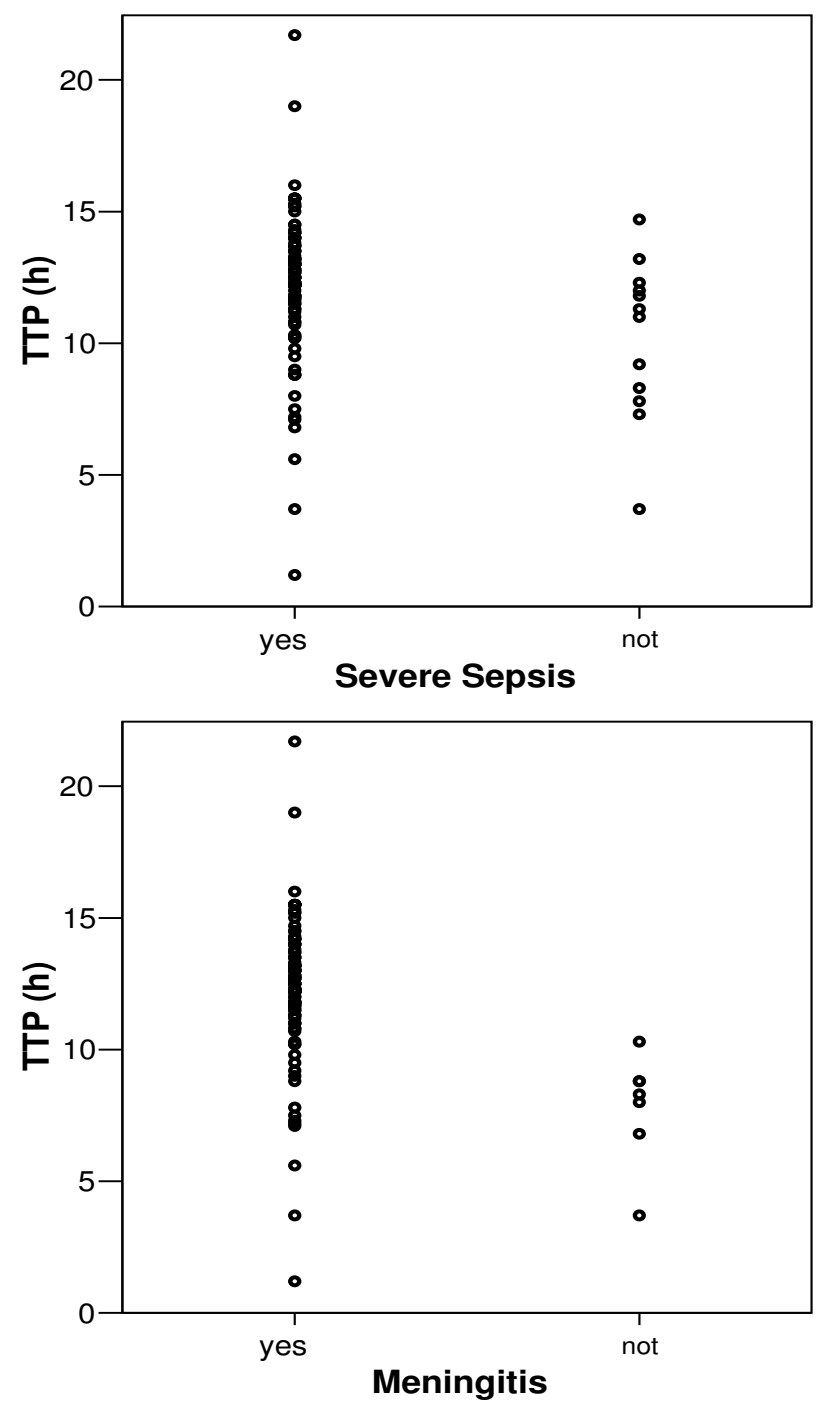

Figure 3

Comparative representation of TTP in patients with and without severe sepsis and meningitis.

fact that only one of the patients who died had been admitted to ICU. The other patients with a fatal outcome had not been admitted to ICU because of a calamitous basal condition, which probably contributed to the fatal outcome. On the other hand the first cause of death was bilateral pneumonia, a process that was not associated with a shorter TTP in our patients.

Our study is retrospective and the results must be interpreted cautiously, though they are supported by several previous investigations. In vitro experiments on simulated blood cultures with the new automated blood culture systems have found a relationship between TTP and bacterial $[9,10]$ and Candida spp. blood concentration [18]. In children, bacterial blood concentration correlates the progno-

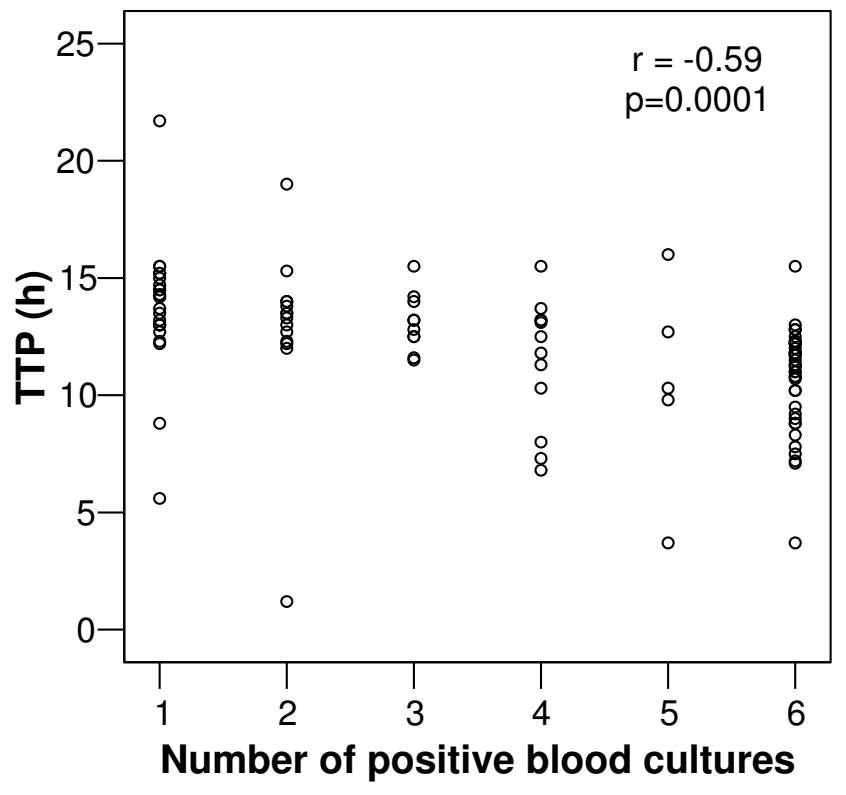

Figure 4

Correlation of the TTP and the number of positive blood cultures of each episode of pneumococcal bacteremia. Two patients with extreme values with only positive one blood culture are excluded ( $120 \mathrm{~h}$ and $\mathrm{I} 22 \mathrm{~h}$ respectively).

sis and the appearance of meningitis, not only in cases of pneumococcal bacteremia $[11,14]$ but also in cases of Haemophilus influence [11-15] and Neisseria meningitidis bacteremia [12]. A correlation also exists between the number of organisms in the CSF and in blood in patients with bacterial meningitis [16]. As TTP is determined by the bacterial blood concentration, it has been suggested as a surrogate marker for the initial bacterial density $[10,19,20][21]$, so it is logical to think that TTP should be in relation to several clinical parameters. In fact TTP is longer when the microorganisms isolated in blood cultures are contaminants in patients with sickle cell disease [22], and in children [23]. A recent study concluded that TTP in patients with Staphylococcus aureus bacteremia is an independent predictor of an endovascular source of infection, metastatic infection or extended bacteremia [24].

Recently, it has also been proved that the value of the difference between the TTP of blood cultures drawn through the central venous catheter and the TTP of those drawn from the peripheral vein is highly diagnostic of catheterrelated bloodstream infection in patients with long-term catheters $[19,20,25]$. It has been proposed that this parameter could substitute the differential quantitative cultures used previously in the diagnosis of catheter related infection $[19,20,24,25]$. 
Table 2: Comparison of characteristics of patients with TTP in the I $^{\text {st }}$ and $4^{\text {th }}$ quartile.

\begin{tabular}{|c|c|c|c|c|}
\hline Characteristic & I stTTP quartile & $2^{\text {th }}$ and $3^{\text {rd }}$ TTP quartiles & $4^{\text {th }}$ TTP quartile & $\mathbf{p}^{*}$ \\
\hline Total (n) & 26 & 54 & 25 & \\
\hline TTP median (interquartile range) & $8.8(7.18-10.2)$ & $12.4(11.8-13)$ & $23.94(\mid 4.2-15.5)$ & \\
\hline \multicolumn{5}{|l|}{ Basal characteristics } \\
\hline Age (years) & $68.5 \pm 18.4$ & $64.6 \pm 18.3$ & $67.32 \pm 17.62$ & $>0.1$ \\
\hline Male $n(\%)$ & $14(53.8)$ & $33(61.1)$ & $14(56)$ & $>0.1$ \\
\hline Time evolution (days) & $3.6 \pm 2.8$ & $3.4 \pm 3.3$ & $3.7 \pm 3.4$ & $>0.1$ \\
\hline Hospitalized n (\%) & $26(100)$ & $46(88.5)$ & $20(80)$ & 0.02 \\
\hline Previous antibiotic n (\%) & $3(11.5)$ & $4(7.4)$ & $2(8)$ & $>0.1$ \\
\hline \multicolumn{5}{|l|}{ Comorbidities } \\
\hline Alcoholism n (\%) & $\mathrm{I}(3.8)$ & II (20.4) & $5(20)$ & $>0.1$ \\
\hline COPD n (\%) & $6(23.1)$ & $12(22.2)$ & $13(52)$ & 0.03 \\
\hline Diabetes n (\%) & $2(7.7)$ & $8(14.8)$ & $\mathrm{I}(4.0)$ & $>0.1$ \\
\hline Neoplasm n (\%) & 0 & $3(5.6)$ & $2(8)$ & $>0.1$ \\
\hline Immunosuppression n (\%) & $2(7.7)$ & $2(3.7)$ & I (4) & $>0.1$ \\
\hline Dementia $\mathrm{n}(\%)$ & $\mathrm{I}(3.8)$ & $3(5.6)$ & $2(8)$ & $>0.1$ \\
\hline \multicolumn{5}{|l|}{ Clinical presentation } \\
\hline $\mathrm{Ta}\left({ }^{\circ} \mathrm{C}\right)$ & $38.3 \pm 0.7$ & $37.9 \pm 1$ & $37.8 \pm 0.8$ & $>0.1$ \\
\hline Chills n (\%) & $7(26.9)$ & $18(33.3)$ & $9(36)$ & $>0.1$ \\
\hline Confusion n (\%) & $9(34.6)$ & $6(11.1)$ & $4(16)$ & 0.08 \\
\hline Severe sepsis or shock n (\%) & $5(19.2)$ & $6(11.1)$ & I (4) & 0.09 \\
\hline Bilateral pneumonia $\mathrm{n}(\%)$ & $3(11.5)$ & $5(9.3)$ & $5(20)$ & $>0.1$ \\
\hline \multicolumn{5}{|l|}{ Origin of bacteremia } \\
\hline Meningitis n (\%) & $7(26.9)$ & 0 & 0 & $<0.001$ \\
\hline Pneumonia n (\%) & $17(65.4)$ & $45(79.6)$ & $23(92)$ & 0.02 \\
\hline Others n (\%) & $2(7.7)$ & $8(14.8)$ & $3(12)$ & $>0.1$ \\
\hline \multicolumn{5}{|l|}{ Laboratory parameters } \\
\hline Penicillin susceptible $\mathrm{n}(\%)$ & $21(80.8)$ & $45(83.3)$ & $19(76)$ & $>0.1$ \\
\hline WBC (cells $/ \mu \mathrm{L})$ & $15880 \pm 5120$ & $19070 \pm 12926$ & $19633 \pm 10812$ & 0.01 \\
\hline Neutrophils (\%) & $89.7 \pm 6.06$ & $78.6 \pm 15.8$ & $85.4 \pm 10.2$ & 0.06 \\
\hline Platelets (cells/ $\mu \mathrm{L})$ & $211580 \pm 64385$ & $188340 \pm 76700$ & $234000 \pm 116387$ & 0.03 \\
\hline Creatinine level (mg/dL) & $1.3 \pm 0.6$ & $1.1 \pm 0.5$ & $1.3 \pm 1$ & $>0.1$ \\
\hline Albumin (mg/dL) & $2.8 \pm 0.7$ & $3 \pm 0.6$ & $3 \pm 0.6$ & $>0.1$ \\
\hline Cholesterol (mg/dL) & $|3| \pm 56.5$ & $139.6 \pm 57.7$ & $177 \pm 53.3$ & $>0.1$ \\
\hline Positive blood cultures ( $\mathrm{n}$ ) & $5 \pm 1.56$ & $4 \pm 2$ & $2 \pm 1.4$ & $<0.001$ \\
\hline \multicolumn{5}{|l|}{ Outcome } \\
\hline Hospital stay (days) & $17.3 \pm 12.8$ & $23.64 \pm 71.6$ & $11.4 \pm 7.9$ & 0.07 \\
\hline Exitus $\mathrm{n}(\%)$ & $4(15.4)$ & $5(9.3)$ & $4(16)$ & $>0.1$ \\
\hline ICU n (\%) & $9(34.6)$ & $5(9.3)$ & 0 & $<0.001$ \\
\hline
\end{tabular}

*P values are referred to the comparisons between patients in the $\left.\right|^{\text {st }}$ and the in $4^{\text {th }}$ quartile TTP

The effect of several factors other than bacterial concentration on TTP has been also addressed. The influence of the different automated blood culture systems on TTP is well documented [26-28]. Also, there are great differences in TTP between different microorganisms in the clinical setting $[22,26-28]$. One factor that can alter TTP is the presence of antibiotic. A recent study has evaluated the effect of various antibiotics on TTP, detecting differences in their prolonging effect depending on the blood culture system used [29]. We have not found differences in TTP among patients treated with antibiotics when blood cultures we performed. Several explanations can be provide for this finding. It is possible that antibiotic treatment was administered earlier to the more seriously ill subgroup of patients. On the other hand the low plasmatic concentrations achieved by a some oral antibiotic treatment could have an negligible effect on TTP. This aspect could be of special relevance in the case of the macrolides in view of the low plasmatic concentrations of this antibiotics [30].

Previous studies focusing on TTP in patients with Streptococcus pneumoniae bacteremia have found similar mean TTP values to ours $[8,26-29]$. However, the only study that analyzed the relationship of TTP with clinical parameters in children with Streptococcus pneumoniae did not find any [8]. Several factors may explain these dissimilar results, such as, for example, the study population (paediatric in their study versus adult population in ours), the number of blood cultures per patient (one versus three), the volume of blood per blood culture bottle (one to three millilitres versus ten millilitres) and the blood culture system used (Bactec versus BacT/Alert) [8]. Other factors not con- 
trolled, such as an erratic delay in placing blood culture bottles in the automated blood culture system after extraction and the variability in the quantity of the blood drawn inoculated in culture bottles may have contributed to the different results.

Another point of interest in our patients is that TTP also has an inverse relationship with the number of positive blood cultures. As has been said, in simulated blood cultures when bacterial blood concentration increases, TTP decreases $[9,10]$, and when bacterial charge increases, the probability of positive blood cultures also increases [9]. If both TTP and the number of positive blood cultures depend on bacterial concentration, their relationship, can be expected, as we have found. This supports the importance of bacterial concentration as a determinant of TTP in patients.

Our study has several limitations. Due to its retrospective design data are obtained from patients' records and some data could be missed. In addition, time waiting to culture after extraction and volume of blood inoculated in blood cultured bottles could influence the TTP and were not recorded. Other limitation is the low number of patients with some of the analyzed characteristics which impede obtain solid conclusions about the relations of TTP with them. Moreover the population studied had a low mortality and a relatively low incidence of comorbidity so the extrapolation of our data to other different populations with higher mortality and degree of immunosuppression should me made with caution. The influence of the blood culture system used in the TTP values also complicate comparisons among different institutions.

Our data support the relationship of TTP with several clinical parameters in patients with Streptococcus pneumoniae bacteremia, although their clinical usefulness probably is limited in view of the narrow spread of their values. However the presence of a low TTP in a patient with Streptococcus pneumoniae bacteremia could alert, for example, for the presence of a meningitis or immunosuppression in patients without a severe sepsis. The refinement of TTP data, including the control of potential influencing factors such as time awaiting culture, blood volume per culture bottle, and perhaps prior antibiotic administration could even improve its relationship with clinical parameters in patients with Streptococcus pneumonia bacteremia.

\section{Conclusion}

Our data support the relationship of TTP with several clinical parameters in patients with Streptococcus pneumoniae bacteremia, and its potential usefulness as a surrogate marker of outcome.

\section{Competing interests}

The author(s) declare that they have no competing interests.

\section{Authors' contributions}

GP conceived the study, participated in the design, the acquisition, analysis and interpretation of data, and performed the statistical analysis.

MJRL participated in the design of the study and its coordination and analysis of data.

JCG participated in the acquisition, analysis and interpretation of data.

LA participated in the acquisition, analysis and interpretation of data.

MPR carried out the microbiological studies, and participated in the coordination, acquisition, analysis and interpretation of data.

\section{Acknowledgements}

We are grateful to Gilles Petty for help in the preparation of the manuscript.

The authors have no conflict of interest or financial support for this work.

\section{References}

I. Musher DM: Streptococcus pneumoniae. In Principles and practice of infectious diseases Edited by: Mandell GL, Bennet JE, Dolin R. Philadelphia, Elsevier-Churchill Livingstone; 2005:2392-24II.

2. Plouffe JF, Breiman RF, Facklam RR: Bacteremia with Streptococcus pneumoniae. Implications for therapy and prevention. Franklin County Pneumonia Study Group. JAMA 1996, 275:194-8.

3. Castillo EM, Rickman LS, Brodine SK, Ledbetter EK, Kelly C: Streptococcus pneumoniae: bacteremia in an era of penicillin resistance. Am J Infect Control 2000, 28:239-43.

4. Laurichesse H, Romaszko JP, Nguyen LT, Souweine B, Poirier V, GuoIon D, Andre M, Ruivard M, De Champs C, Caillaud D, Labbe A, Beytout J: Clinical characteristics and outcome of patients with invasive pneumococcal disease, Puy-de-Dome, France, 1994-1 998. Eur J Clin Microbiol Infect Dis 2001, 20:299-308.

5. Maugein J, Guillemot D, Dupont MJ, Fosse T, Laurans G, Roussel-Delvallez $M$, Thierry J, Vergnaud M, Weber M, Poirier B: Clinical and microbiological epidemiology of Streptococcus pneumoniae bacteremia in eight French counties. Clin Microbiol Infect 2003, 9:280-8.

6. Farinas-Alvarez C, Farinas MC, Garcia-Palomo JD, Gonzalez-Ruiz M, Fernandez-Mazarrasa C, Parra JA, Gonzalez-Macias J: Prognostic factors for pneumococcal bacteremia in a university hospital. Eur J Clin Microbiol Infect Dis 2000, 19:733-4I.

7. Marfin AA, Sporrer J, Moore PS, Siefkin AD: Risk factors for adverse outcome in persons with pneumococcal pneumonia. Chest 1995, 107:457-62.

8. Neuman MI, Harper MB: Time to positivity of blood cultures for children with Streptococcus pneumoniae bacteremia. Clin Infect Dis 200I, 33: I324-8.

9. Haimi-Cohen Y, Vellozzi EM, Rubin LG: Initial concentration of Staphylococcus epidermidis in simulated pediatric blood cultures correlates with time to positive results with the automated, continuously monitored BACTEC blood culture system. J Clin Microbiol 2002, 40:898-901.

10. Blot F, Schmidt E, Nitenberg G, Tancrede C, Leclercq B, Laplanche A, Andremont A: Earlier positivity of central-venous- versus 
peripheral-blood cultures is highly predictive of catheterrelated sepsis. J Clin Microbiol 1998, 36:105-9.

II. Sullivan TD, LaScolea LJ, Neter E: Relationship between the magnitude of bacteremia in children and the clinical disease. Pediatrics 1982, 69:699-702.

12. Sullivan TD, LaScolea LJ Jr: Neisseria meningitidis bacteremia in children: quantitation of bacteremia and spontaneous clinical recovery without antibiotic therapy. Pediatrics 1987, 80:63-7.

13. Santosham M, Moxon ER: Detection and quantitation of bacteremia in childhood. J Pediatr 1977, $91: 719-21$.

14. Bell LM, Alpert G, Campos JM, Plotkin SA: Routine quantitative blood cultures in children with Haemophilus influenzae or Streptococcus pneumoniae bacteremia. Pediatrics 1985, 76:90I-4.

15. Marshall GS, Bell LM: Correlates of high grade and low grade Haemophilus influenzae bacteremia. Pediatr Infect Dis J 1988 , 7:86-90.

16. La Scolea LJ Jr, Dryja D: Quantitation of bacteria in cerebrospinal fluid and blood of children with meningitis and its diagnostic significance. J Clin Microbiol 1984, 19:187-90.

17. Bone RC, Balk RA, Cerra FB, Dellinger RP, Fein AM, Knaus WA, Schein RM, Sibbald W]: Definitions for sepsis and organ failure and guidelines for the use of innovative therapies in sepsis. The ACCPISCCM Consensus Conference Committee. American College of Chest Physicians/Society of Critical Care Medicine. Chest 1992, 101:1644-55.

18. George BJ, Horvath LL, Hospenthal DR: Effect of inoculum size on detection of Candida growth by the BACTEC 9240 automated blood culture system using aerobic and anaerobic media. / Clin Microbiol 2005, 43:433-5.

19. Blot F, Nitenberg G, Chachaty E, Raynard B, Germann N, Antoun S, Laplanche A, Brun-Buisson C, Tancrede C: Diagnosis of catheterrelated bacteraemia: a prospective comparison of the time to positivity of hub-blood versus peripheral-blood cultures. Lancet 1999, 354:107I-77.

20. Malgrange VB, Escande MC, Theobald S: Validity of earlier positivity of central venous blood cultures in comparison with peripheral blood cultures for diagnosing catheter-related bacteremia in cancer patients. J Clin Microbiol 200I, 39:274-8.

21. Vigano EF, Vasconi E, Agrappi C, Clerici P: Use of simulated blood cultures for time to detection comparison between BacT/ ALERT and BACTEC 9240 blood culture systems. Diagn Microbiol Infect Dis 2002, 44:235-40.

22. Norris CF, Smith-Whitley K, McGowan KL: Positive blood cultures in sickle cell disease: time to positivity and clinical outcome. J Pediatr Hematol Oncol 2003, 5:390-5.

23. Raad I, Hanna HA, Alakech B, Chatzinikolaou I, Johnson MM, Tarrand J: Differential time to positivity: a useful method for diagnosing catheter-related bloodstream infections. Ann Intern Med 2004, 1 40: 18-25.

24. Khatib R, Riederer K, Saeed S, Johnson LB, Fakih MG, Sharma M, Tabriz MS, Khosrovaneh A: Time to positivity in Staphylococcus aureus bacteremia: possible correlation with the source and outcome of infection. Clin Infect Dis 2005, 41:594-8.

25. Jorgensen $\mathrm{JH}$, Mirrett S, McDonald LC, Murray PR, Weinstein MP, Fune J, Trippy CW, Masterson M, Reller LB: Controlled clinical laboratory comparison of BACTEC plus aerobic/F resin medium with BacT/Alert aerobic FAN medium for detection of bacteremia and fungemia. J Clin Microbiol 1997, 35:53-8.

26. Cockerill FR 3rd, Reed GS, Hughes JG, Torgerson CA, Vetter EA Harmsen WS, Dale JC, Roberts GD, Ilstrup DM, Henry NK: Clinical comparison of BACTEC 9240 plus aerobic/F resin bottles and the isolator aerobic culture system for detection of bloodstream infections. J Clin Microbiol 1997, 35: 1469-72.

27. Murray PR, Hollick GE, Jerris RC, Wilson ML: Multicenter comparison of BACTEC 9050 and BACTEC 9240 blood culture systems. J Clin Microbiol 1998, 36:1601-3.

28. Alpern ER, Alessandrini EA, Bell LM, Shaw KN, McGowan KL: Occult bacteremia from a pediatric emergency department: current prevalence, time to detection, and outcome. Pediatrics 2000, 106:505-II.

29. Vigano EF, Vasconi E, Agrappi C, Clerici P, Melloni P: Use of simulated blood cultures for antibiotic effect on time to detection of the two blood culture systems BacT/ALERT and BACTEC 9240. New Microbiol 2004, 27:235-48.
30. Kelley MA, Weber DJ, Gilligan P, Cohen MS: Breakthrough pneumococcal bacteremia in patients being treated with azithromycin and clarithromycin. Clin Infect Dis 2000, 31:1008-II.

\section{Pre-publication history}

The pre-publication history for this paper can be accessed here:

http://www.biomedcentral.com/1471-2334/6/79/prepub
Publish with Bio Med Central and every scientist can read your work free of charge

"BioMed Central will be the most significant development for disseminating the results of biomedical research in our lifetime. "

Sir Paul Nurse, Cancer Research UK

Your research papers will be:

- available free of charge to the entire biomedical community

- peer reviewed and published immediately upon acceptance

- cited in PubMed and archived on PubMed Central

- yours - you keep the copyright

Submit your manuscript here:

http://www.biomedcentral.com/info/publishing_adv.asp
BioMedcentral 